\title{
BMJ Open COVID-19 seroprevalence among hospital staff and preprocedural patients in Thai community hospitals: a cross-sectional study
}

\author{
Tanawin Nopsopon (D) , ${ }^{1,2}$ Krit Pongpirul (D) , ${ }^{1,3,4,5}$ Korn Chotirosniramit, ${ }^{1}$ \\ Narin Hiransuthikul (1) ${ }^{1}$
}

To cite: Nopsopon T,

Pongpirul K, Chotirosniramit K, et al. COVID-19 seroprevalence among hospital staff and preprocedural patients in Thai community hospitals: a crosssectional study. BMJ Open 2021;11:e046676. doi:10.1136/ bmjopen-2020-046676

- Prepublication history and additional supplemental material for this paper are available online. To view these files, please visit the journal online (http://dx.doi.org/10.1136/ bmjopen-2020-046676).

Received 05 November 2020 Accepted 13 October 2021

D) Check for updates

(c) Author(s) (or their employer(s)) 2021. Re-use permitted under CC BY-NC. No commercial re-use. See rights and permissions. Published by BMJ.

For numbered affiliations see end of article.

Correspondence to Associate Professor Dr Krit Pongpirul;

doctorkrit@gmail.com

\section{ABSTRACT}

Objectives We aimed to explore the seroprevalence of hospital staff comparing to preprocedural patients in Thai community hospitals to shed light on the situation of COVID-19 infection of frontline healthcare workers in low infection rate countries where mass screening was not readily available.

Design Cross-sectional study.

Setting 52 community hospitals in 35 provinces covered all regions of Thailand.

Participants 857 participants consisted of 675 hospital staff and 182 preprocedural patients.

Outcome measure COVID-19 seroprevalence using a locally developed rapid IgM/lgG test kit

Results Overall, $5.5 \%$ of the participants (47 of 857 ) had positive $\operatorname{lgM}, 0.2 \%$ (2 of 857 ) had positive $\lg G$ which both of them also had positive lgM. Hospitals located in the central part of Thailand had the highest IgM seroprevalence (11.9\%). Preprocedural patients had a higher rate of positive IgM than the hospital staff $(12.1 \%$ vs $3.7 \%$ ). Participants with present upper respiratory tract symptoms had a higher rate of positive IgM than those without $(9.6 \%$ vs $4.5 \%)$. Three quarters $(80.5 \%, 690$ of $857)$ of the participants were asymptomatic, of which, 31 had positive $\operatorname{lgM}(4.5 \%)$ which consisted of 20 of 566 healthcare workers (3.5\%) and 11 of 124 preprocedural patients (8.9\%).

Conclusions COVID-19 antibody test could detect a substantial number of potential silent spreaders in Thai community hospitals where the nasopharyngeal PCR was not readily available, and the antigen test was prohibited. Antibody testing should be encouraged for mass screening in a limited resource setting, especially in asymptomatic individuals.

Trial registration TCTR20200426002.

\section{INTRODUCTION}

PCR was introduced as a diagnostic test of choice for COVID-19 infection. However, it might not be readily available or affordable in many facilities and could pose an unnecessary risk to the healthcare providers during the specimen collection. Besides, a recent study raised a concern of false-negative results from
Strengths and limitations of this study

- This study covered all regions in Thailand and consisted of community hospitals from 35 out of 77 provinces.

- We used a locally developed IgM/lgG test kit with high internal validation to shed light on the actual COVID-19 situation in areas in which nasopharyngeal PCR testing was not readily available.

- This study provided a real-life experience to gather crucial information despite restricted resources.

- We did not have a chance to perform the serological test among the COVID-19 confirmed cases as the mild case had to get quarantined and the moderate and severe ones were referred to a higher level of care, which could affect the seroprevalence.

- We could not perform multiple serological tests at different time points as doing so was not approved by the ethics committee.

the nasopharyngeal PCR test for SARS-CoV-2 in patients with high pretest probability and encouraged the development of a highly sensitive test. ${ }^{1}$

Antibody testing provides additional information for epidemic investigation and control with high sensitivity and simplicity, especially when used along with the nasopharyngeal PCR test. At an early stage of the COVID-19 pandemic, antibody testing was used for mass screening to identify and track the missing silent spreaders in Singapore. ${ }^{2}$ Asymptomatic patients are considered to be one of the important sources of COVID-19 transmission, ${ }^{3}$ with approximately one-fifth transmission rate to close contact individuals. ${ }^{4}$ Additionally, there was a $13 \%$ estimated proportion of asymptomatic patients with COVID-19 in general and 37\% in healthcare providers. $^{5}$

While the nasopharyngeal PCR test was considered gold-standard, there were increasing studies that reported both PCR 
and antibody test results. In the early phase of pandemic, there was a study in China reported a $2.5 \%$ overall COVID-19 seroprevalence in the hospital setting with subgroup analysis of $1.8 \%$ in healthcare workers and $3.5 \%$ in asymptomatic patients. ${ }^{6}$ Recent meta-analysis reported $8 \%$ SARS-CoV-2 seroprevalence in healthcare workers before vaccine initiation. ${ }^{7}$

An early study on the development of SARS-CoV-2 antibodies in symptomatic patients with COVID-19 reported that IgM had the highest value during 20-22 days after onset while IgG had the highest value during 17-19 days after onset. ${ }^{8}$ More complete information on immunoglobulin development was reported in a recent systematic review that IgM had median seroconversion time between four to 14 days, reached its peak at $2-5$ weeks, then declined to an undetectable level at 6 weeks postonset while $\operatorname{IgG}$ had median seroconversion time between 12 and 15 days, reached its peak at 3-7 weeks, then diminished after 8 weeks after onset. ${ }^{9}$ Since SARS-CoV-2 infectivity was likely to diminish after 8-13 days postonset, ${ }^{10}$ antibody tests for IgM seroprevalence might not suit for early diagnosis of current COVID-19 cases with infectivity, but would be more appropriate to support the diagnosis of recent COVID-19 infection after 2 weeks of onset. ${ }^{11}$

Preferably, utilisation of both nasopharyngeal PCR and antibody tests would provide an accurate estimation of the COVID-19 situation in a specific area. However, there were several low-income to middle-income countries that could not pay for the cost of nasopharyngeal PCR tests and had to restrict the eligibility for PCR testing to a limited population for the optimisation of resource usage. In Thailand, the nasopharyngeal PCR test was offered in suspected individuals with strict criteria during the initial phase of the COVID-19 pandemic. As a more feasible, cheaper and safer alternative to the nasopharyngeal PCR in a limited resource setting, the antibody test is not only useful for an epidemiological investigation but could also be used for mass screening of potential silent spreaders-asymptomatic COVID-19 individuals. ${ }^{2}$

Hospital is one of the best venues for getting and spreading pathogens. There are two types of people in the hospital who potentially are silent spreaders and need antibody testing: (1) healthcare workers who have a relatively higher risk of infection than laypersons and (2) asymptomatic patients who need procedural treatment or operation but do not meet the criteria for nasopharyngeal PCR testing.

\section{METHODS}

\section{Study population}

From 8 April to 26 June 2020, hospital staff and patients who needed procedural treatment or operation visiting the hospital during the recruiting period and did not meet the national nasopharyngeal PCR testing criteria in 244 hospitals (215 community hospitals and 29 general hospitals) from all regions of Thailand were recruited for antibody testing in their community hospital. Hospitals included in the study came from a national survey about willingness to use antibody testing for COVID-19 screening. Of 215 community hospitals, data from 52 hospitals $(24.2 \%)$ in 35 provinces from all regions which could be considered representative of community hospitals across Thailand were readily available for the analysis performed on 29 June 2020. Participants with active symptoms meeting national criteria for nasopharyngeal PCR testing were quarantined and excluded. Participants were asked to answer a questionnaire about risk history for COVID-19, recent symptoms within the past 2 weeks, and previous nasopharyngeal PCR test results if available.

National criteria for nasopharyngeal PCR testing for COVID-19 National criteria for nasopharyngeal PCR testing for COVID-19 for symptomatic layperson during the study period included fever with one of the upper respiratory infection symptoms (cough, rhinitis, sore throat, anosmia, tachypnoea or dyspnoea) and one of risk history (history of travel to a high-risk area, an occupation involving tourists, crowded place, or contact with many people, history of going to crowded place in the community, or history of close contact confirmed COVID-19 case) within 14 days before the onset of symptoms. For symptomatic healthcare workers, the criteria were less strict. Either fever or one of the upper respiratory infection symptoms was sufficient for PCR testing. During recruiting period, there was almost impossible for an asymptomatic person to get PCR tested.

\section{Antibody testing}

Baiya Rapid COVID-19 IgG/IgM test kit (Baiya Phytopharm, Thailand) which reports the presence of IgM and IgG qualitatively using lateral flow immunoassay technique and receptor-binding protein of spike protein of SARS-CoV-2 for antigen, was used in this study free of charge. The internal validation of the test kit using the serum of 51 nasopharyngeal PCR confirmed COVID-19 cases and 150 controls showed sensitivity $94.1 \%$ (48 of 51) and specificity $98.0 \%$ (147 of 150) for IgM or IgG antibody. Of 51 PCR confirmed COVID-19 cases, 56.9\% (29 of 51 ) were $\operatorname{IgM}+\operatorname{IgG}-, 37.3 \%$ (19 of 51) were $\operatorname{IgM}+$ IgG+, $5.9 \%$ (3 of 51 ) were IgM-IgG-. No IgM- IgG + was detected in 51 PCR confirmed COVID-19 cases from interval validation. Participants with positive $\operatorname{IgM}$ were encouraged to have a nasopharyngeal PCR test if available. There was no hospital or centre readily available for neutralising antibody testing during the study period.

\section{Age-adjusted seroprevalence}

PCR confirmed COVID-19 cases were obtained from the Thailand government report on 30 June 2020. Seroprevalence data were presented as unadjusted seroprevalence and compared with direct age-adjusted seroprevalence using combined participating population, Thailand population, and world population provided by the WHO for 2000-2025 population. 


\section{Statistical analysis}

Categorical data were presented with counts and percentages while continuous data were provided with median and IQRs. The 95\% CI of the seroprevalence was calculated by Wilson's method using binomial probabilities. Correlation between seroprevalence and PCR confirmed COVID-19 prevalence was tested using Spearman's correlation. Missing data were excluded. A two-tailed $\mathrm{p}<0.05$ was considered statistically significant. All data were analysed using Stata V.16.1.

\section{Patient and public involvement}

The research question and outcome measure were developed from both patient and public involvement. For patient involvement, visiting a hospital must not harm patients; however, the unknown status of COVID-19 infection in the pandemic period led to a doubtable situation of no harm. For public involvement, the national policy about COVID-19 test at the study period made unstable situation among the public, a national survey was conducted to identify the situation in the community and general hospitals to find places that should be the priority for this study to provide more confidence situation for hospital visiting and working. Patients and hospital staff who participated in this study had a report for their serology status, and the results of this study would be disseminated not only to participants but also to the public via an online channel to provide evidence of the actual situation.

\section{RESULTS}

\section{Community hospital demographic}

Overall, 52 community hospitals from $46.1 \%$ of provinces in Thailand ( 35 of 76 ) which consisted of $58.2 \%$ of national population (35416545 of 60892671 ) participated in this study. Participation rates varied across regions-Northeastern (55\%), Central (50.0\%), Southern (42.9\%), Northern $(33.3 \%)$ and Eastern $(28.6 \%)$ (online supplementary table E1).

\section{Participant demographic}

From 52 community hospitals, 857 participants which consisted of 675 hospital staff and 182 preprocedural patients were included in the study. Their median age was 37 years (IQR 27-45), $74.7 \%$ were female, $98.8 \%$ were Thai and $80.5 \%$ were asymptomatic. The most common symptoms were cough $(9.7 \%)$, rhinitis $(7.5 \%)$, sore throat $(6.4 \%)$, fever $(5.7 \%)$ and dyspnoea (3.5\%). History of travel to the high-risk area was $6.0 \%$, history of close contact to the confirmed COVID-19 case was $15.4 \%$, and $14.5 \%$ had nasopharyngeal PCR negative (table 1 ). Forty-seven participants (5.5\%, 95\% CI 4.1 to 7.2$)$ had IgM antibodies against SARS-CoV-2 whereas the IgG antibody was found in two participants $(0.2 \%, 95 \%$ CI 0.1 to 0.8). Participants from the Central region of Thailand had the highest IgM seroprevalence $(11.9 \%$, 95\% CI 8.4 to 16.5), while the Northern region had the lowest seroprevalence $(1.6 \%, 95 \%$ CI 0.3 to 8.7$)$ (figure 1$)$.

\section{Age-adjusted IgM seroprevalence}

Age-adjusted IgM seroprevalence with combined participating population showed almost similar results with unadjusted IgM seroprevalence. However, age-adjusted seroprevalence using Thailand population showed increasing seropositive rate in Thailand from $5.5 \%$ to $6.3 \%$, Central region from $11.9 \%$ to $15.3 \%$ and Northern region from $1.6 \%$ to $1.8 \%$, while decreasing seroprevalence in Northeastern region from $3.0 \%$ to $1.6 \%$, Eastern region from $4.0 \%$ to $2.8 \%$ and Southern region from $1.8 \%$ to $1.5 \%$. Adjusted with the world standard population from the WHO (2000-2025) showed decreasing trends of seroprevalence both overall and in most regions (table 2).

\section{Participant characteristics and seroprevalence}

Preprocedural patients had an unexpectedly higher proportion of positive IgM than the hospital staff $(12.1 \%$ vs $3.7 \%$ ), especially patients in the Central region of Thailand $(27.9 \%, 95 \%$ CI 18.2 to 40.2$)$ while patients in the Northern and Southern regions showed zero seroprevalences. Also, hospital staff in the Central region had the highest seroprevalence $(6.6 \%, 95 \%$ CI 3.8 to 11.1$)$ while those in the Northern region had the lowest $(1.7 \%$, 95\% CI 0.3 to 9.0). Overall, the seropositive prevalence was not different between males and females $(5.8 \%$ vs $5.5 \%)$. Paradoxically, the seroprevalences were higher in participants without a history of travel to a high-risk area $(5.6 \%$ vs $3.9 \%)$ and those without a history of close contact to confirmed COVID-19 case $(5.7 \%$ vs $4.5 \%$ ) than their counterparts. The same paradox also applied to preprocedural patients. Patients without travel history were likely to have an antibody for SARS-CoV-2 (13.9\% vs $3.2 \%$ ) and patients without close contact to the case also had more chance to develop an antibody $(12.3 \%$ vs $9.1 \%)$. However, healthcare workers with travel history had slightly more chance to develop IgM $(5.0 \%$ vs $3.7 \%)$ and with close contact history ( $4.1 \%$ vs $3.6 \%)$. In general, participants with upper respiratory tract symptoms had a higher chance of being seropositive $(9.6 \%$ vs $4.5 \%)$, of which dyspnoea had the highest $(30.0 \%, 95 \%$ CI 16.7 to 47.9). Likewise, preprocedural patients with dyspnoea had the most IgM positive $(29.6 \%, 95 \%$ CI 15.9 to 48.5$)$ and healthcare workers with dyspnoea $(33.3 \%, 95 \%$ CI 6.1 to 79.2). Of 690 participants without present upper respiratory tract symptoms, 31 had IgM positive for COVID-19 $(4.5 \%, 95 \%$ CI 3.2 to 6.3$)$ which consisted of 20 of 566 healthcare workers $(3.5 \%, 95 \%$ CI 2.3 to 5.4$)$ and 11 of 124 patients $(8.9 \%, 95 \%$ CI 5.0 to 15.2$)$. History of negative nasopharyngeal PCR was associated with a surprisingly higher chance of seropositive than those with no PCR test result ( $6.5 \%$ vs $5.3 \%$ ) (table 3 ). Unfortunately, none of the participants with positive IgM had opportunities for nasopharyngeal PCR testing. 
Table 1 Demographic details of participants

\begin{tabular}{|c|c|c|c|}
\hline & $\begin{array}{l}\text { All participants } \\
\mathrm{N}(\%)\end{array}$ & $\begin{array}{l}\text { Hospital staff } \\
\text { n (\%) }\end{array}$ & $\begin{array}{l}\text { Preprocedural patients } \\
\text { n (\%) }\end{array}$ \\
\hline Total & 857 & 675 & 182 \\
\hline Median age, years (25th-75th percentile) & $37(27-45)$ & $36.5(28-45)$ & $37(25-53)$ \\
\hline Male & $207(24.1 \%)$ & $145(21.5 \%)$ & $62(34.1 \%)$ \\
\hline Female & $640(74.7 \%)$ & $521(77.2 \%)$ & $119(65.4 \%)$ \\
\hline Unspecified & $10(1.2 \%)$ & $9(1.3 \%)$ & $1(0.5 \%)$ \\
\hline Thai & $847(98.8 \%)$ & $671(99.4 \%)$ & $176(96.7 \%)$ \\
\hline Non-Thai & $10(1.2 \%)$ & $4(0.6 \%)$ & $6(3.3 \%)$ \\
\hline \multicolumn{4}{|l|}{ Region } \\
\hline North & $61(7.1 \%)$ & $59(8.8 \%)$ & $2(1.1 \%)$ \\
\hline Northeast & $269(31.4 \%)$ & $220(32.6 \%)$ & $49(26.9 \%)$ \\
\hline Central & 244 (28.5\%) & 183 (27.1\%) & $61(33.5 \%)$ \\
\hline South & $109(12.7 \%)$ & $88(13.0 \%)$ & $21(11.6 \%)$ \\
\hline East & $174(20.3 \%)$ & $125(18.5 \%)$ & $49(26.9 \%)$ \\
\hline \multicolumn{4}{|l|}{ History of travel to high-risk area } \\
\hline Yes & $51(6.0 \%)$ & $20(3.0 \%)$ & $31(17.0 \%)$ \\
\hline No & 806 (94.0\%) & $655(97.0 \%)$ & $151(83.0 \%)$ \\
\hline \multicolumn{4}{|l|}{ History of close contact confirmed case } \\
\hline Yes & $132(15.4 \%)$ & $121(17.9 \%)$ & $11(6.0 \%)$ \\
\hline No & $725(84.6 \%)$ & $554(82.1 \%)$ & $171(94.0 \%)$ \\
\hline Asymptomatic & $690(80.5 \%)$ & $566(83.9 \%)$ & $124(68.1 \%)$ \\
\hline Symptomatic & $167(19.5 \%)$ & $109(16.1 \%)$ & $58(31.9 \%)$ \\
\hline Fever & $49(5.7 \%)$ & $17(2.5 \%)$ & $32(17.6 \%)$ \\
\hline Cough & $83(9.7 \%)$ & $52(7.7 \%)$ & $31(17.0 \%)$ \\
\hline Rhinitis & $64(7.5 \%)$ & $47(7.0 \%)$ & $17(9.3 \%)$ \\
\hline Sore throat & $55(6.4 \%)$ & $37(5.5 \%)$ & $18(9.9 \%)$ \\
\hline Dyspnoea & $30(3.5 \%)$ & $3(0.4 \%)$ & $27(14.8 \%)$ \\
\hline \multicolumn{4}{|l|}{ Previous nasopharyngeal PCR status } \\
\hline Negative & $124(14.5 \%)$ & $77(11.4 \%)$ & $47(25.8 \%)$ \\
\hline Never tested & 733 (85.5\%) & 598 (88.6\%) & 135 (74.2\%) \\
\hline
\end{tabular}

Data were presented in counts and percentages unless otherwise specified.

\section{COVID-19 prevalence and IgM seroprevalence}

PCR confirmed COVID-19 case and population data were acquired for participating provinces. Overall, COVID-19 prevalence was 2.44 cases per 100000 population. Participating provinces in the Eastern region had the highest prevalence of COVID-19 (6.54 cases per 100000 population) while provinces in the Northeastern had the lowest prevalence (supplemental table E2). There was no correlation between IgM seroprevalence, and PCR confirmed COVID-19 prevalence $(\mathrm{p}=0.199)$.

\section{Characteristics of IgG seropositive participants}

IgG was detected in two participants $(0.2 \%, 95 \%$ CI 0.1 to 0.8 ) who also had a positive IgM antibody. In other words, we did not find any participants with isolated positive IgG. Participant A was a Thai female healthcare worker who worked in a community hospital in the Central region of Thailand. She had a sore throat but had no history of travel to a high-risk area or close contact to a confirmed COVID-19 case and did not have a nasopharyngeal PCR test before. Participant B was a Thai female preprocedural patient who visited another community hospital in the Central region. She had no symptoms, no history of travel to a high-risk area, or close contact to confirmed the COVID-19 case. Participant B had a previously negative nasopharyngeal PCR result (table 4).

\section{DISCUSSION}

During an early phase of the pandemic in Thailand, which had approximately 61 million inhabitants located in the South-East Asia region, there were $5.5 \%$ overall estimated 


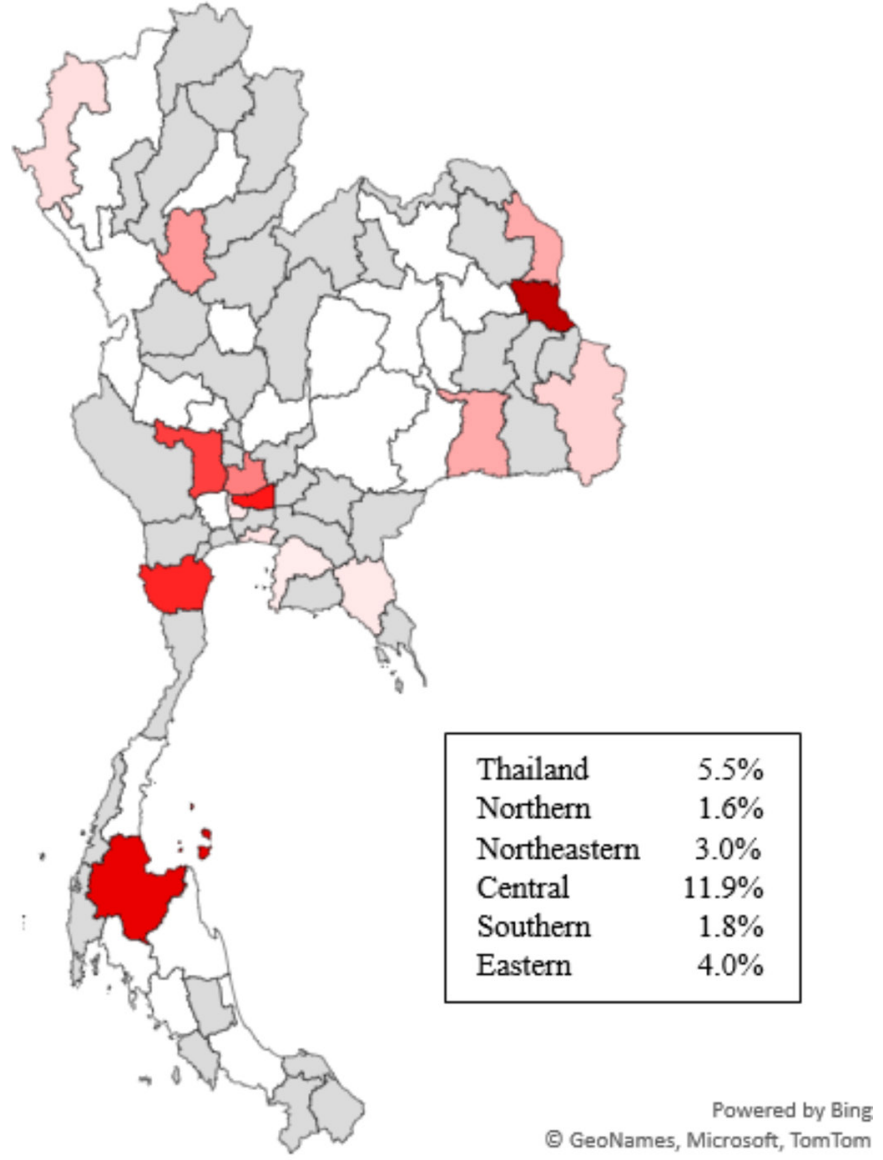

Seroprevalence (\%)

$$
0 \quad 50 \quad 100
$$

Figure 1 Unadjusted IgM seroprevalence of hospital staffs and preprocedural patients in community hospitals across geographical regions of Thailand. Seroprevalence was scaled into colour gradient which white region represented the lowest seroprevalence and dark red region represented the highest seroprevalence. Grey region represented region that was not participated in the study.
IgM and $0.2 \%$ overall IgG seroprevalence in community hospital staffs and preprocedural patients. Overall IgM seroprevalence was highest in the community hospitals located in the central region of Thailand. Preprocedural patients showed a higher estimated IgM seroprevalence than the healthcare workers $(12.1 \%$ vs $3.7 \%)$. Among asymptomatic participants accounted for approximately $80 \%$ of participants, the overall estimated IgM seroprevalence was $4.5 \%$ which could be subcategorised to $3.5 \%$ in healthcare workers and $8.9 \%$ in preprocedural patients. Additionally, participants with present upper respiratory tract symptoms had a higher rate of positive IgM at $9.6 \%$.

COVID-19 seroprevalence in asymptomatic staff and patients in Thai community hospitals was higher than in hospitals in China $(4.5 \%$ vs $2.5 \%) .{ }^{6}$ Seroprevalence in asymptomatic hospital staff in Thailand was also higher than hospitals in China (3.5\% vs $1.8 \%),{ }^{6}$ but less than a tertiary hospital in Belgium (3.5\% vs $6.4 \%) .{ }^{12}$ Moreover, seroprevalence in asymptomatic hospital staff in Thailand community hospital was higher than asymptomatic hospital staffs in a Thai provincial hospital $(3.5 \%$ vs $0.7 \%),{ }^{13}$ but was less than asymptomatic frontline firefighters/paramedics of US fire department $(3.5 \%$ vs $7.7 \%) .{ }^{14}$ Asymptomatic patients in Thailand seemed to have higher seroprevalence than in China $(8.9 \%$ vs $3.5 \%){ }^{6}$ Unlike China and Belgium where the seroprevalences were mostly from positive $\mathrm{IgG}$, our study revealed mostly positive IgM. The possible explanation would be different study periods among studies that represented the different stages of epidemic in the regions. Comparison with a Belgium hospital should be interpreted with caution due to the unknown nasopharyngeal PCR status of Belgium subjects.

Most seropositive participants in this study had positive IgM only while only a few had both positive IgM and IgG. We conjecture three possible explanations which might relate to the dominance of IgM positive results. First, the relation of antibody testing timepoint and the onset of COVID-19 might play a significant contribution. ${ }^{15}$ Although both IgM and

Table 2 Unadjusted and age-adjusted IgM seroprevalence in community hospitals across geographical regions of Thailand

\begin{tabular}{|c|c|c|c|c|}
\hline Regions & $\begin{array}{l}\text { Unadjusted IgM } \\
\text { seroprevalence (\%) }\end{array}$ & $\begin{array}{l}\text { Age-adjusted IgM } \\
\text { seroprevalence with } \\
\text { combined participating } \\
\text { population (\%) }\end{array}$ & $\begin{array}{l}\text { Age-adjusted IgM } \\
\text { seroprevalence with } \\
\text { Thailand population (\%) }\end{array}$ & $\begin{array}{l}\text { Age- } \\
\text { adjusted IgM } \\
\text { seroprevalence } \\
\text { with world } \\
\text { standard } \\
\text { population (\%) }\end{array}$ \\
\hline Thailand* & 5.5 & NA & 6.3 & 5.1 \\
\hline Central $^{\star}$ & 11.9 & 11.9 & 15.3 & 12.2 \\
\hline Southern & 1.8 & 2.4 & 1.5 & 1.2 \\
\hline Eastern & 4.0 & 3.9 & 2.8 & 2.6 \\
\hline
\end{tabular}

*Not include Bangkok which has no community hospital.

NA, not available. 
Table 3 Demographic characteristics and seroprevalence in hospital staff and preprocedural patients

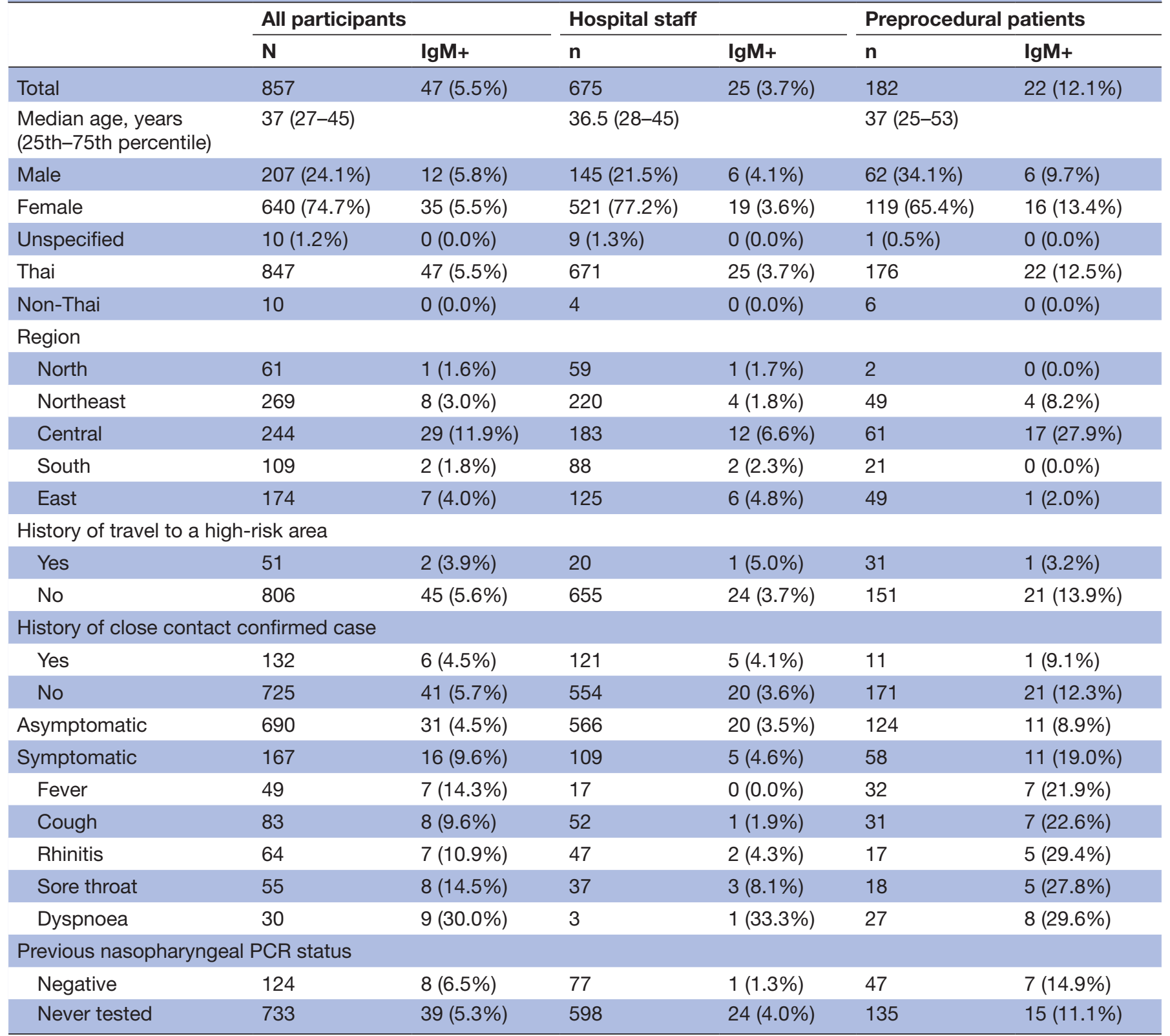

Data were presented in counts and percentages unless otherwise specified.

NA, not available.

IgG could develop to be detectable in some patients during the first-week post-onset, ${ }^{16}$ approximately $70 \%$ of symptomatic patients developed IgM to detectable level during the second week after onset and raised to $90 \%$ of total antibody test positive by days $11-24$ postonset. ${ }^{17}$ Regards the immunoglobulin level, COVID-19 confirmed cases usually had higher IgM levels than IgG during the first 2 weeks after onset with a reversal afterward. ${ }^{18}$ The study period was an early stage of the pandemic in Thailand, thus the participants with recent COVID-19 infection might not develop detectable IgG yet, thus lead to a false-negative result of the IgG test. ${ }^{19}$ Second, there was heterogeneity regards antibody development since some patients with COVID-19 might have impregnable innate immunity and recovered from the disease without developing any antibody. ${ }^{20}$ Third, the exclusion criteria of our observational study excluded all confirmed COVID-19 cases who had a higher chance of developed IgG.

Since the study was based on a hospital setting, the IgM seroprevalence in the study might be an overestimation of the general population due to the higher risk of COVID-19 infection in hospitals, ${ }^{21}$ especially in the Central region of Thailand where the high population density might associate with the increasing transmission. ${ }^{22} 23$ On the other hand, the antibody test for IgM might underestimate the true prevalence of COVID-19 infection in the hospital setting, particularly those who were in the infectious stage since the IgM seroprevalence was more effective to support the diagnosis of recent COVID-19 infection 
after 2 weeks of onset, ${ }^{11}$ while the infectivity was likely to plummet during the first and second week of onset. ${ }^{10}$

There was a very high proportion of participants who had been contacted with COVID-19 confirmed cases. This situation was not unexpected because the participants who had contact with the confirmed case might seek medical care and nasopharyngeal PCR test, but they were not eligible for the PCR test due to national policy during the study period required patients to be both symptomatic and had a high risk of exposure to COVID-19 to get tested. However, people with a history of close contact to confirmed COVID-19 cases or travel to a high-risk area turned out to have a low COVID-19 seroprevalence in our study. One possible explanation could be the successful implementation of the national nasopharyngeal PCRbased screening policy, low barrier to medical care under the Universal Coverage scheme as well as disease awareness and health literacy of the Thais. People with those predefined risks might have already sought medical attention; several cases might have been identified with the PCR test and were either quarantined or sent to proper care. The remaining eligible individuals might be dominated by those without the predefined risks.

To prevent nosocomial spread in a community hospital or primary hospital setting, hospital staff should be tested for COVID-19 before working their routine and should repeat the test at a specified period. Antibody testing was less uncomfortable than nasopharyngeal PCR testing which might lead to more compliance when hospital staff was planned to get multiple tests. False-negative could occur in any test. High sensitivity testing was encouraged for high-risk populations including hospital staff to reduce false negatives. ${ }^{1}$ Negative result on nasopharyngeal PCR with negative antibody test or IgG positive only might be acceptable for hospital staff starting the work when such highly sensitive test was not completely developed. To help prevent community spread in an aspect of a community hospital setting, patients who visited the hospital with any primary symptom should be tested with rapid antibody testing to screen some potential spreader and prevent them from returning to the community during spreadable period especially when nasopharyngeal PCR testing was not readily available in a remote area.

Serological testing provides some crucial epidemiological information and would have been more effective when combined with other diagnostic tests such as nasopharyngeal PCR or rapid antigenic tests. However, there was a unique situation in Thailand during the study period in which the eligibility criteria for nasopharyngeal swabs for PCR test were very strict and those who should have been tested mostly did not meet the strict criteria. Moreover, the rapid antigenic tests were not approved in Thailand until July 2021. While all participants with positive results from the free-of-charge rapid IgM/IgG test provided in this study were encouraged to get nasopharyngeal PCR testing, a majority of community hospitals still did not have access to the PCR testing because of both financial and non-financial reasons. The recommendation for 
nasopharyngeal PCR testing after positive rapid IgM/IgG test was not fully complied so we did not have information about the participants with positive IgM. However, with the immunoglobulin status and PCR results, we can shape the situation more accurately for both individual and regional views. Hopefully, with this and other vigorous and dedicated studies on antibody status around the globe, serology testing would provide useful information for pandemic control.

Given the fact that IgM represents a recent infection while IgG is suggestive of past infection, excluding COVID-19 confirmed cases who had a higher chance of developing IgG during the study period would underestimate the actual IgG seroprevalence and underestimate the actual proportion of seropositive participants who had previous nasopharyngeal PCR testing. While this might be considered a limitation of our study as compared with other studies that did not exclude COVID-19 confirmed cases, our observation reflected a real picture of seroprevalence in a country with low COVID-19 incidence and mortality. We did not have a chance to perform the serological test among the COVID-19 confirmed cases as the mild case had to get quarantined and the moderate and severe ones were referred to a higher level of care. Also, we could not perform multiple serological tests at different time points as doing so was not approved by the ethics committee.

Only 2 out of 47 IgM-positive participants also had IgGpositive and none of the IgM-positive participants got subsequent nasopharyngeal PCR test after IgM-positive status. There was a possibility that IgM positive might be false positive. However, the false-positive rate for IgM or IgG was 2\% according to internal validation of the test kit while IgM positive seroprevalence was $5.5 \%$. While the false positive of IgM might occur due to imperfection of test kit and no confirmation with PCR test after positive antibody against COVID-19 which might lead to overestimation of actual IgM seroprevalence, this limitation shaped the real-world situation in an early phase of pandemic awareness in a community hospital setting where more advanced test methods were not readily available.

\section{CONCLUSIONS}

COVID-19 antibody test could detect a substantial number of potential silent spreaders in Thai community hospitals where the nasopharyngeal PCR was not readily available, and the antigen test was prohibited. Antibody testing should be encouraged for mass screening in a limited resource setting, especially in asymptomatic individuals.

\section{Author affiliations}

${ }^{1}$ Department of Preventive and Social Medicine, Chulalongkorn University Faculty of Medicine, Bangkok, Thailand

${ }^{2}$ Department of Epidemiology, Harvard T.H. Chan School of Public Health, Boston, Massachusetts, USA

${ }^{3}$ School of Global Health, Chulalongkorn University Faculty of Medicine, Bangkok, Thailand
${ }^{4}$ Department of International Health and Department of Health, Behavior and Society, Johns Hopkins Bloomberg School of Public Health, Baltimore, Maryland, USA

${ }^{5}$ Bumrungrad International Hospital, Bangkok, Thailand

\section{Twitter Krit Pongpirul @doctorkrit}

AcknowledgmentsWe thank Baiya Phytopharm, Thailand for supporting the Baiya Rapid COVID-19 lgG/lgM test kit. The company did not involve in the data analysis, interpretation and manuscript preparation.

Contributors TN conceptualised the study, design the methodology, administrated the project, contributed to data curation and clinical investigation, validate the data, performed formal analysis and provided software, visualised the data and wrote the original draft of the manuscript. KP conceptualised the study, design the methodology, administrated the project, contributed to data curation and clinical investigation, validate the data, performed formal analysis and provided software, provided resources for the study, supervised the study and wrote the original draft of the manuscript. KC contributed to data curation and clinical investigation, and validate the data. $\mathrm{NH}$ conceptualised the study, design the methodology, administrated the project, provided resources for the study and supervised the study. All authors reviewed, edited the final manuscript, agreed to be held accountable for all aspects of the work related to its accuracy and integrity. KP acts as the guarantor of this work.

Funding The authors have not declared a specific grant for this research from any funding agency in the public, commercial or not-for-profit sectors.

Map disclaimer The inclusion of any map (including the depiction of any boundaries therein), or of any geographic or locational reference, does not imply the expression of any opinion whatsoever on the part of BMJ concerning the legal status of any country, territory, jurisdiction or area or of its authorities. Any such expression remains solely that of the relevant source and is not endorsed by BMJ. Maps are provided without any warranty of any kind, either express or implied.

Competing interests None declared.

Patient and public involvement statement Patient and/or the public were involved in the design, or conduct, or reporting, or dissemination plans of this research. Refer to the Methods section for further details.

Patient consent for publication Consent obtained directly from patient(s).

Ethics approval Ethics approval was obtained from the Institutional Review Board of Chulalongkorn University (IRB No. 236/63), Ethics Committee of National Cancer Institute Thailand (EC No. 015/2563), Research Ethics Committee of Nan Hospital (REC No. 063/2563), Ethics Committee of Chaophraya Yommarat Hospital (YM No. 013/2563), Institutional Review Board of Songkhla Hospital (IRB No. 2020-Md-J3-04001), Ethics Committee of Lampang Hospital (EC No. 26/63), Ethics Committee of Sikhio Hospital (SK No. 2020/001), Ethics Committee of Samut Sakhon Hospital, Research Ethics Committee of Prapokklao Hospital (CTIREC No. 030/63), Institutional Review Board of Burapha University (HS No. 027/2563), Ethics Committee of Srinakharinwirot University (SWUEC No. 119/2563F), Institutional Review Board of Phaholpolpayuhasena Hospital (IRB No. 2020-04), Institutional Review Board of Samitivej Sukhumvit Hospital (IRB No. 001/2563), Research Ethics Committee of Pattani Provincial Public Health Office (RECPTN No. 015/63), Institutional Review Board of Bangplee Hospital (IRB No. 1/2563), Institutional Review Board of Chonburi Hospital (IRB No. 08/2563), Institutional Review Board of Surin Hospital (IRB No. 15/2563), Institutional Review Board of Child and Adolescent Mental Health Rajanagarindra Institute (IRB No. 2563/005) and Institutional Review Board of Sukhothai Hospital (IRB No. 44/2563). Given no ethics committees are available in the participating community hospitals, participation in this study was approved by the hospital directors or representatives. All participants provided written informed consent including consent for publication of raw data.

Provenance and peer review Not commissioned; externally peer reviewed.

Data availability statement All data relevant to the study are included in the article or uploaded as supplementary information. All data relevant to the study are included in the article; the anonymized dataset is uploaded as a Supporting Information file.

Supplemental material This content has been supplied by the author(s). It has not been vetted by BMJ Publishing Group Limited (BMJ) and may not have been peer-reviewed. Any opinions or recommendations discussed are solely those of the author(s) and are not endorsed by BMJ. BMJ disclaims all liability and responsibility arising from any reliance placed on the content. Where the content includes any translated material, BMJ does not warrant the accuracy and reliability 
of the translations (including but not limited to local regulations, clinical guidelines, terminology, drug names and drug dosages), and is not responsible for any error and/or omissions arising from translation and adaptation or otherwise.

Open access This is an open access article distributed in accordance with the Creative Commons Attribution Non Commercial (CC BY-NC 4.0) license, which permits others to distribute, remix, adapt, build upon this work non-commercially, and license their derivative works on different terms, provided the original work is properly cited, appropriate credit is given, any changes made indicated, and the use is non-commercial. See: http://creativecommons.org/licenses/by-nc/4.0/.

\section{ORCID iDs}

Tanawin Nopsopon http://orcid.org/0000-0003-0428-973X

Krit Pongpirul http://orcid.org/0000-0003-3818-9761

Narin Hiransuthikul http://orcid.org/0000-0002-1517-7445

\section{REFERENCES}

1 Woloshin S, Patel N, Kesselheim AS. False Negative Tests for SARS-CoV-2 Infection - Challenges and Implications. N Engl J Med 2020;383:e38.

2 Yong SEF, Anderson DE, Wei WE. Connecting clusters of COVID-19: an epidemiological and serological investigation. Lancet Infect Dis 2020;20:809-15.

3 Kronbichler A, Kresse D, Yoon S, et al. Asymptomatic patients as a source of COVID-19 infections: a systematic review and metaanalysis. Int J Infect Dis 2020;98:180-6.

4 Yanes-Lane M, Winters N, Fregonese F, et al. Proportion of asymptomatic infection among COVID-19 positive persons and their transmission potential: a systematic review and meta-analysis. PLoS One 2020;15:e0241536

5 Chen C, Zhu C, Yan D, et al. The epidemiological and radiographical characteristics of asymptomatic infections with the novel coronavirus (COVID-19): a systematic review and meta-analysis. Int J Infect Dis 2021;104:458-64.

6 Xu X, Sun J, Nie S. Seroprevalence of immunoglobulin M and G antibodies against SARS-CoV-2 in China. Nat Med 2020.

7 Kayı İlker, Madran B, Keske Siran, et al. The seroprevalence of SARS-CoV-2 antibodies among health care workers before the era of vaccination: a systematic review and meta-analysis. Clin Microbiol Infect 2021;27:1242-1249.
8 Long Q-X, Liu B-Z, Deng H-J, et al. Antibody responses to SARSCoV-2 in patients with COVID-19. Nat Med 2020;26:845-8.

9 Post N, Eddy D, Huntley C, et al. Antibody response to SARSCoV-2 infection in humans: a systematic review. PLoS One 2020;15:e0244126.

10 Walsh KA, Jordan K, Clyne B, et al. SARS-CoV-2 detection, viral load and infectivity over the course of an infection. $J$ Infect 2020;81:357-71.

11 Chen M, Qin R, Jiang M, et al. Clinical applications of detecting lgG, IgM or IgA antibody for the diagnosis of COVID-19: a meta-analysis and systematic review. Int J Infect Dis 2021;104:415-22.

12 Steensels D, Oris E, Coninx L, et al. Hospital-Wide SARS-CoV-2 antibody screening in 3056 staff in a tertiary center in Belgium. JAMA 2020;324:195.

13 Nopsopon T, Pongpirul K, Chotirosniramit K, et al. Seroprevalence of hospital staff in a Province with zero COVID-19 cases. PLoS One 2021;16:e0238088.

14 Caban-Martinez AJ, Schaefer-Solle N, Santiago K, et al. Epidemiology of SARS-CoV-2 antibodies among firefighters/ paramedics of a US fire department: a cross-sectional study. Occup Environ Med 2020;77:857-61.

15 Sethuraman N, Jeremiah SS, Ryo A. Interpreting diagnostic tests for SARS-CoV-2. JAMA 2020;323:2249-51.

$16 \mathrm{Ma} \mathrm{H}$, Zeng $\mathrm{W}, \mathrm{He} \mathrm{H}$, et al. Serum IgA, IgM, and IgG responses in COVID-19. Cell Mol Immunol 2020;17:773-5.

17 Beeching NJ, Fletcher TE, Beadsworth MBJ. Covid-19: testing times. BMJ 2020;369:m1403.

18 Qin X, Shen J, Dai E, et al. The seroprevalence and kinetics of IgM and IgG in the progression of COVID-19. BMC Immunol 2021;22:14.

19 Younes N, Al-Sadeq DW, AL-Jighefee H, et al. Challenges in laboratory diagnosis of the novel coronavirus SARS-CoV-2. Viruses 2020:12:1. doi:10.3390/v12060582

20 Wang $B$, Wang L, Kong X, et al. Long-term coexistence of SARSCoV-2 with antibody response in COVID-19 patients. J Med Virol 2020;92:1684-9.

21 Van Praet JT, Claeys B, Coene A-S, et al. Prevention of nosocomial COVID-19: another challenge of the pandemic. Infect Control Hosp Epidemiol 2020;41:1355-6.

22 Bhadra A, Mukherjee A, Sarkar K. Impact of population density on Covid-19 infected and mortality rate in India. Model Earth Syst Environ 2020:623-9.

23 Yin $\mathrm{H}$, Sun T, Yao L, et al. Association between population density and infection rate suggests the importance of social distancing and travel restriction in reducing the COVID-19 pandemic. Environ SCI Pollut Res Int 2021;28:1-7. doi:10.1007/s11356-021-12364-4 